\title{
The Possible Radio Protective Role of Gum Arabic on The Kidney Cortex of Adult Male Albino Rats Heba Ahmed Mohamed Kandeal ${ }^{1}$, Fatma Ahmed Eid, Hemmat Mansour Abdelhafez ${ }^{1}$, Amr Mahmoud Abd El-Hady ${ }^{2}$, \\ ${ }^{1}$ Zoology Department, Faculty of Science, Al-Azhar University, Egypt \\ ${ }^{2}$ Faculty of Applied Medical Sciences, Misr University for Science and Technology (MUST), Egypt
}

\begin{abstract}
Aim of the work: the use of ionizing radiation exposure increases, oxidative stress especially for cancer patients. Therefore, there is a critical need to develop antioxidants that prevent oxidative stress damage. Gum Arabic is an antioxidant and anti-inflammatory mediator. This study aimed to assess the possible radio protective effect of gum Arabic (Acacia senegal) against gamma irradiation-induced injury on the rat kidney. Materials and Methods: four groups of male Albino rats (each of 12 rats) received normal saline (G1), dose 5Gy of gamma irradiation (G2), orally administered with $25 \mathrm{mg} / \mathrm{kg}$ gum Arabic for 3 weeks (G3) and gamma irradiation plus oral gum Arabic administration for 7 days before and 21 days after irradiation (G4). Results: in the gamma irradiated group, histopathological and ultrastructural examinations of the rat kidney cortical tissue revealed signs of degeneration to the cortical renal tubules and glomeruli. In contrast, gum Arabic treatment alleviated most of the damaging effects of gamma radiation.

Conclusion: oral administration of gum Arabic could ameliorate adverse gamma radiation-induced effects that might be attributed to its antioxidative and free radical scavenging effects.
\end{abstract}

Keywords: gamma radiation, kidney, gum Arabic, radio protective, rats.

\section{INTRODUCTION}

Ionizing radiation (IR) exposure is a worldwide (global) problem. Everyone can be exposed to IR under several conditions - at home, at workplaces, as a patient or caregiver in the medical settings and other sources. Its medical application for diagnosis and therapy is one of the increasing activities. Gamma and X-rays are the most commonly used types of IR in medical the settings ${ }^{(1)}$. Living cell absorption of IR may lead to some chemical and biological cellular damages through direct or indirect actions ${ }^{(2)}$. Reactive oxygen species (ROS) production is one of the most important damaging effects of IR and the imbalance between excessive ROS generation and cellular endogenous antioxidant defense system production reflects an oxidative stress process ${ }^{(3)}$.Antioxidant treatment is one of the most effective strategies in protecting living cells against radiation exposure damages, where some natural substances obtained from plant sources showed a protective effect against radiation-induced oxidative stress (4).Applications of natural products are considered as an alternative method to ameliorate chronic renal failure disease $^{(5)}$. Gum Arabic (GA) is a natural polysaccharide, extracted from the dried discharges of sticky stems and branches of Acacia trees, mainly Acacia senegal and Acacia seyal trees, with no viscous soluble fiber content ${ }^{(6)}$.GA trees are one of the genus Acacia, subfamily Mimosoideae and family Leguminosae ${ }^{(7)}$.GA was found to be potent in treating patients with chronic renal failure. The nephroprotective mechanism is unclear, but may include antioxidant and anti-inflammatory effects ${ }^{(8)}$.
This study aimed to assess the possible radio protective effect of GA on the kidney of the irradiated adult male Albino rats through the use of some histological and ultrastructural studies.

\section{MATERIAL and METHODS \\ Chemicals}

The gum Arabic powder was obtained from El Gomhouria Company, Cairo, Egypt, it was administrated to the rat groups after suspension in distilled water at a dose of $25 \mathrm{mg} / \mathrm{kg} /$ day using a gastric tube for consecutive 21 days ${ }^{(9)}$.

\section{Radiation treatment}

Whole-body 5-Gy single-dose gamma irradiation process of rats was performed at the National Center for Radiation Research and Technology, Nasr City, Cairo, Egypt, using Cesium-137 Gamma cell 40. The dose rate was $0.62 \mathrm{~Gy} / \mathrm{min}$ at the time of the experiment.

\section{Experimental animals}

Experimental adult male Albino rats (Wistar albino), weighing 160-180 grams at the beginning of the experiment, were purchased from the Egyptian Holding Company for Biological Products and Vaccines, Helwan, Cairo, Egypt. The animals were placed in plastic cages at proper environmental circumstances of light, ventilation, food and water. This experiment was performed in accordance with the guidelines set by the National Institutes of Health (Publication no. 85-23, revised 1996). 


\section{Experimental design}

The animals were arbitrarily categorized into 4 groups (12 rats each), treated in parallel and classified as follows:

Group 1 (C): administrated with distilled water for only 21 days and served as the control rats.

Group 2(R): subjected to 5-Gy single-dose gamma radiation.

Group 3(GA): received GA at $25 \mathrm{mg} / \mathrm{kg}$ body weight as a daily oral dose for 21 days.

Group 4(GA+R): received GA at $25 \mathrm{mg} / \mathrm{kg}$ body weight as a daily oral dose for 7 days before and 21 days after irradiation.

\section{Histological preparations}

After 7 and 21 days of all experimentation, all rats were anesthetized by light diethyl ether and sacrificed rapidly to remove the kidney. The tissue was cut into $3 \mathrm{~mm}^{3}$ pieces, fixed in $10 \%$ neutral buffered formalin fixative and embedded in paraffin wax. Section cutting was performed at $5 \mu \mathrm{m}$ thickness and stained with Harris hematoxylin and eosin and Mallory's trichrome for the histopathological study ${ }^{(\mathbf{1 0})}$.

\section{Electron microscopic preparations}

The kidney sections were processed for electron microscopic examination. Thin pieces were fixed with 5\% glutaraldehyde in $0.05 \mathrm{~mol} / \mathrm{L}$ sodium cacodylate buffer for 1 hour, then removed, sliced $1 \mathrm{~mm}^{3}$ thick and placed in the same fixative overnight. Tissue slices were then post fixed in osmium tetraoxide for 2 hours (11), dehydrated and embedded in Epon. One-micron-thick sections were cut and stained with $0.5 \%$ toluidine blue and examined by light microscope. Ultrathin sections were cut and stained with uranyl acetate and lead citrate for ultrastructural examination at the electron microscopy unit of Al-Azhar University.

\section{Ethical approval:}

All the experimental procedures were carried out according to the principles and guidelines of the Ethics Committee of the of Faculty of science, Al-Azhar University, Cairo, Egypt conformed to "Guide for the care and use of Laboratory Animals" for the use and welfare of experimental animals, published by the US National Institutes of Health (NIH publication No. 8523, 1996).

\section{RESULTS}

The kidney cortex of the control rats showed the normal histological appearance of Bowman's capsules, glomeruli, proximal (PCTs) and distal convoluted tubules (DCTs) (Fig. 1). After 7 days of exposure of rats to 5 Gy of gamma radiation their kidneys cortical sections showed highly degenerated, atrophied, or hypertrophied glomeruli with pyknotic and karyolytic nuclei of the cells of the convoluted tubules. Reduced
Bowman's spaces and numerous hemorrhagic areas were also detected in between (Figs. $2 \& 3$ ). On the other hand, cells of the DCTs and PCTs showed pyknotic and karyolytic nuclei with degenerated cuboidal cells (Figs. $4 \& 5)$.

Progressive histopathological changes represented by highly thickened arterial walls with hemolysed blood cells and lymphocytic infiltrations were noted 21 days after gamma radiation exposure (Figs. 6 and 7).

Compared to the control group, the kidney cortex of rats of the GA group showed normal appearance in sections of the kidney cortex tissue after 7(Fig. 8) and 21 days (Fig. 9). The treatment of irradiated rats with GA application showed some histopathological signs of improvement indicated by intact Bowman's capsules, relatively well-preserved glomeruli and convoluted tubules architecture after 7 days (Fig. 10) and 21 days (Fig. 11) of irradiation.

Normal distribution of collagen fibers was detected in the Bowman's capsules, glomeruli, the basement membranes of the convoluted tubules (Fig. 12) in section of the kidney cortex of the control group. Increased collagen fibers with brightly stained red blood cells (RBCs) were detected in the hemorrhagic areas and the congested glomeruli 7 days (Fig. 13) and 21 days (Fig. 14) of irradiation. Compared to the control group, the kidney cortex of rats of the GA group showed normal collagen fibers deposition after 7(Fig. 15) and 21days (Fig. 16) of the treatment. After 7 days of exposure of rats to $5 \mathrm{~Gy}$ of gamma radiation their kidneys cortical sections showed somewhat normal deposition of collagen fibers throughout the kidney cortex tissue after 7 (Fig. 17) and 21 days (Fig. 18) of radiation exposure.

Ultrastructural examination of the kidney cortical zone of the control group revealed normal glomeruli attaining normal glomerular basement membranes, mesangial cells, podocytes and foot processes (Fig. 19). Normal PCTs were detected with numerous microvilli of the brush borders at the apical surfaces of the cells with normal basement membranes, many mitochondria and lysosomes. The nuclei were located more toward the base than the apex. Small vesicles were seen at the base of the microvilli that fuse to form vacuoles at the apical pits of the microvilli (Fig. 20). The DCT had few microvilli and the nuclei were located at the apical surfaces of the cells with a relatively small number of mitochondria mainly toward the basal surface of the cells (Fig. 21). Moreover, 7 days after exposure of the rats to whole body 5-Gy gamma radiation, ultrastructural examination of the kidney cortical sections revealed degeneration in both PCTs and DCTs where the PCTs showed malformed and ruptured brush borders, increased size of lysosomes, disintegrated with electron faint and hardly detected mitochondria and 
atrophied and pyknotic nuclei with irregular nuclear membranes; disintegrated cytoplasmic organoids were detected in numerous necrotic areas with highly increased collagen deposition (Figs.22 -29). The DCTs showed reduced lumens with highly reduced number of their cuboidal cells nuclei and some cells had karyolitic nuclei and increased number of lysosomes with appearance of degenerated areas and degenerated mitochondria and multiple necrotic areas (Figs. 26-28). Furthermore, 21 days after irradiation (Fig. 30) there were thickened processes of podocytes and malformed nuclei of the mesangial cells. The CTs lost their normal architecture because some nuclei of cuboidal cells contained highly atrophied and pyknotic nuclei with condensed chromatin, whereas others contained nuclei with disintegrated and fragmented chromatin. The cortical PCTs showed debris of degenerated cytoplasmic organoids with unclear borders of the cuboidal cells; their nuclei were irregular in shape with numerous lysosomes and degenerated areas (Figs. 31-33). The kidney cortical tissue of GA-administrated rats after 7 (Figs. 34 and 35) and 21 days (Figs. 36 and 37) of treatment exhibited normal capillary tufts that contained RBCs, podocytes with primary and secondary processes and mesangial cells. The PCTs showed details of the microvilli brush borders and numerous mitochondria in the basal part of the cells. The DCTs showed a large number of mitochondria in their cells. The kidney cortex of rats orally treated with GA 1 week after irradiation showed normal architecture of podocytes and mesangial cells in the glomeruli of Bowman's capsules and somewhat normal appearance of nuclei, brush borders and mitochondria of the cuboidal cells of PCTs(Figs. 38 -40). In addition, the kidney cortex of rats orally treated with GA after 21 days showed normal appearance of mesangial and podocyte cells with well-developed cytoplasmic organoids of the cuboidal cells of both DCTs and PCTs (Figs. 41-44).
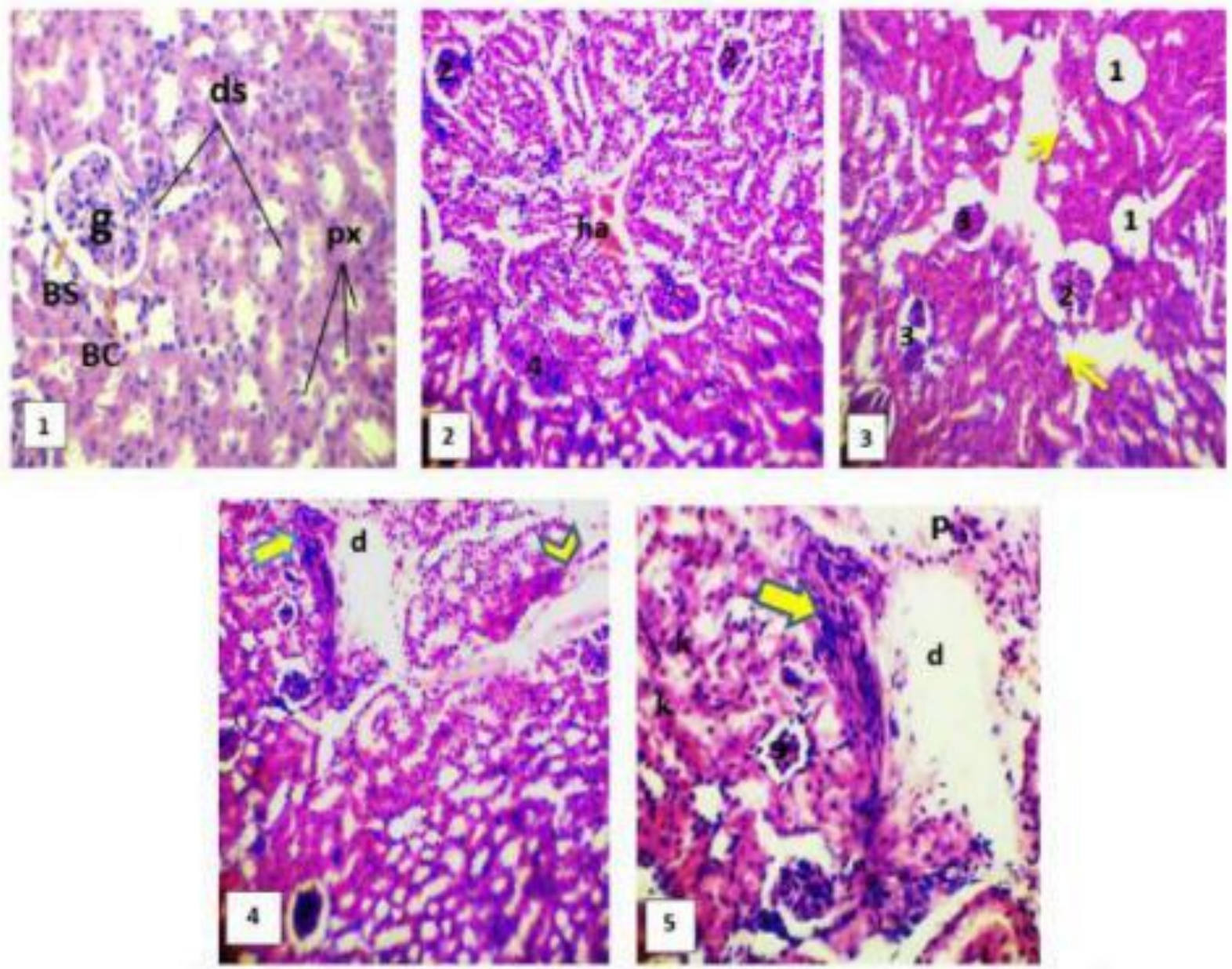

Figure1: a photomicrograph of section in kidney cortex of the control group showing normal Bowman's capsules (BC), Bowman's space (BS), glomeruli (g), and proximal (px) and distal (ds) convoluted tubules. (H\&E X400).

Figures 2-5: photomicrographs of the kidney cortical sections of $R$ groups after 7 days of irradiation.

Figs. 2\&3: Showing highly affected glomeruli; some of them are completely degenerated (1), lobulated (2), atrophied (3), or hypertrophied (4) with reduced Bowman's space. Notice: highly degenerated cuboidal cells of the convoluted tubules $(\rightarrow)$ and hemorrhagic areas (ha). (H\&E X250). Figs. 4\& 5: Showing the distal and proximal convoluted tubules include pyknotic (p) and karyolitic (k) nuclei with lymphocytic infiltration ( $\boldsymbol{\nabla}$ ), numerous degenerated areas (d), and edema $(\rightarrow$. (H\&E, 4 X200\&5 X250). 

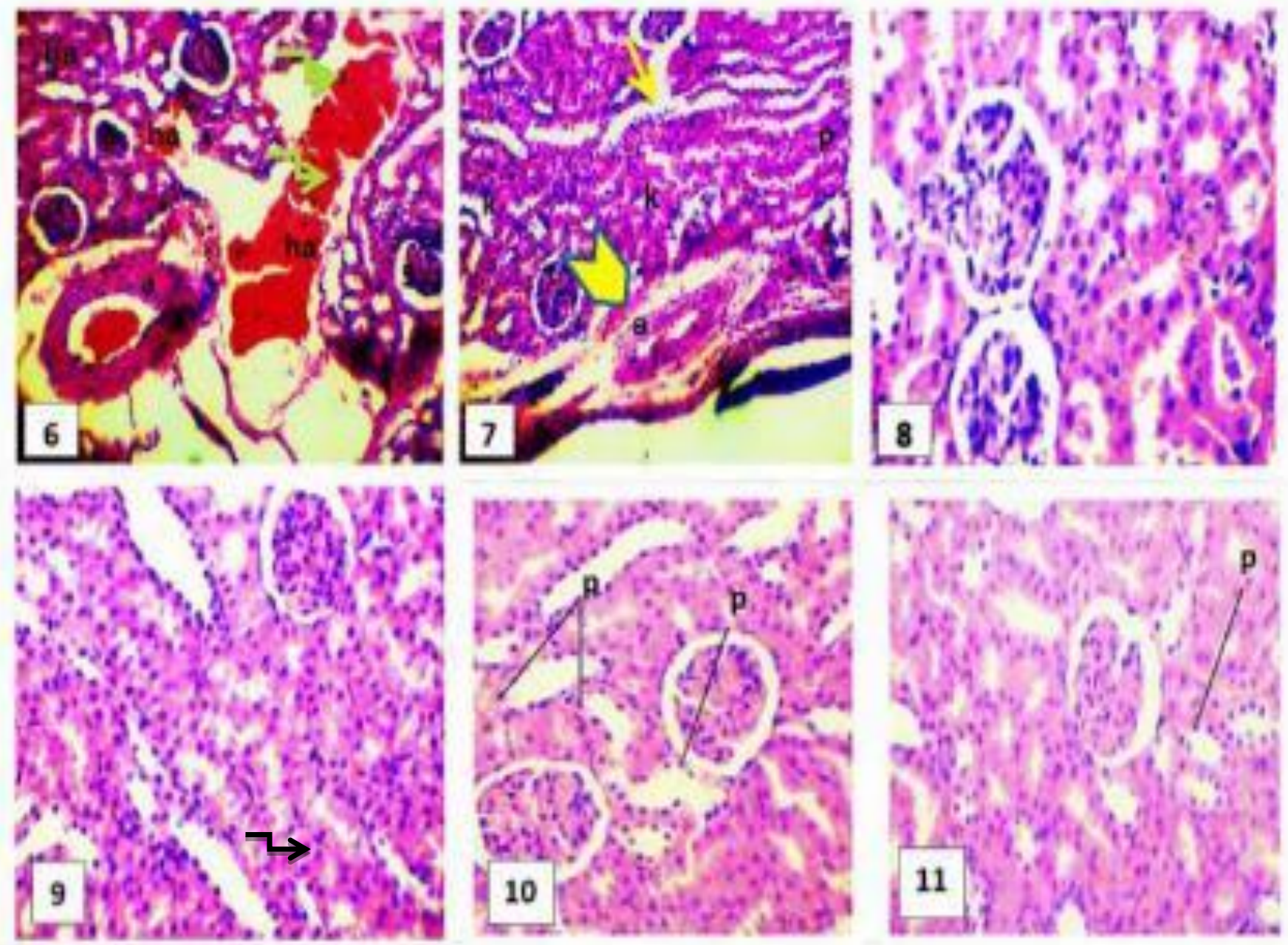

Figures 6\&7: photomicrographs of the kidney cortical sections of R groups after 21 days of irradiation showing numerous hemorrhagic areas (ha) containing hemolysed blood cells, hemosiderin granules $(\longrightarrow)$ and highly thickened, elongated and corrugated arterial wall (a). (H\&E X250).

Figures 8\& 9: showing the kidney cortex tissue of GA groups after 7 (Fig. 8) and 21 days (Fig. 9) of treatment showing normal histological appearance. (H\&E X 400).

Figures. 10\&11: GA+R group after 7 (Fig. 10) and 21days (Fig. 11) showing somewhat histological architecture with appearance of some pyknotic nuclei (p) of cells of the convoluted tubules and lobulated glomeruli. (H\&E X400). 

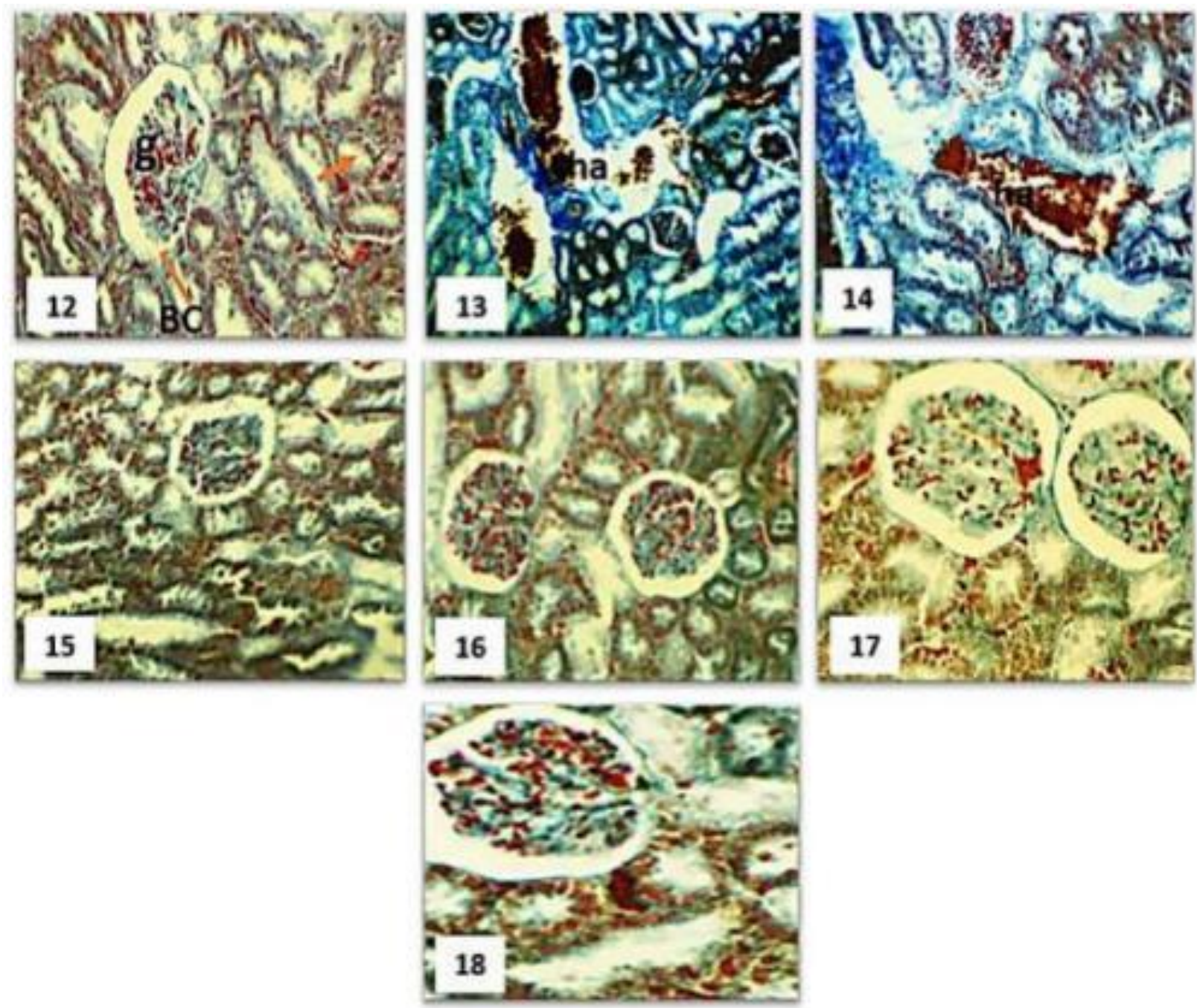

Figure 12: a photomicrograph of section in kidney cortex of the control group showing normal distribution of collagen fibers in the Bowman's capsules (BC), brush borders of the proximal convoluted tubules $(\rightarrow)$, glomeruli $(\mathrm{g})$ and the basement membranes of the convoluted tubules (Mallory's trichrome stain X400).

Figures 13,14: photomicrographs of the kidney cortical sections of R groups after 7 (Fig. 13) and 21 days (Fig. 14) of irradiation showing increased collagen fibers in the intertubular spaces, glomeruli, and convoluted tubules, especially the proximal convoluted one and in the blood vessel walls with numerous brightly red-stained hemorrhagic areas (ha) (Mallory's trichrome stain X250).

Figures15\&16: photomicrographs of kidney cortical sections of GA groups after 7 (Fig. 15) and 21 days (Fig. 16) of treatment showing normal appearance of collagen fibers (Mallory's trichrome stain X400).

Figures17\& 18: photomicrographs of section in the kidney cortex tissue of groups GA+R after 7(Fig. 17) and 21 days (Fig. 18) of irradiation showing somewhat normal appearance of collagen fibers (Mallory's trichrome stain X400). 

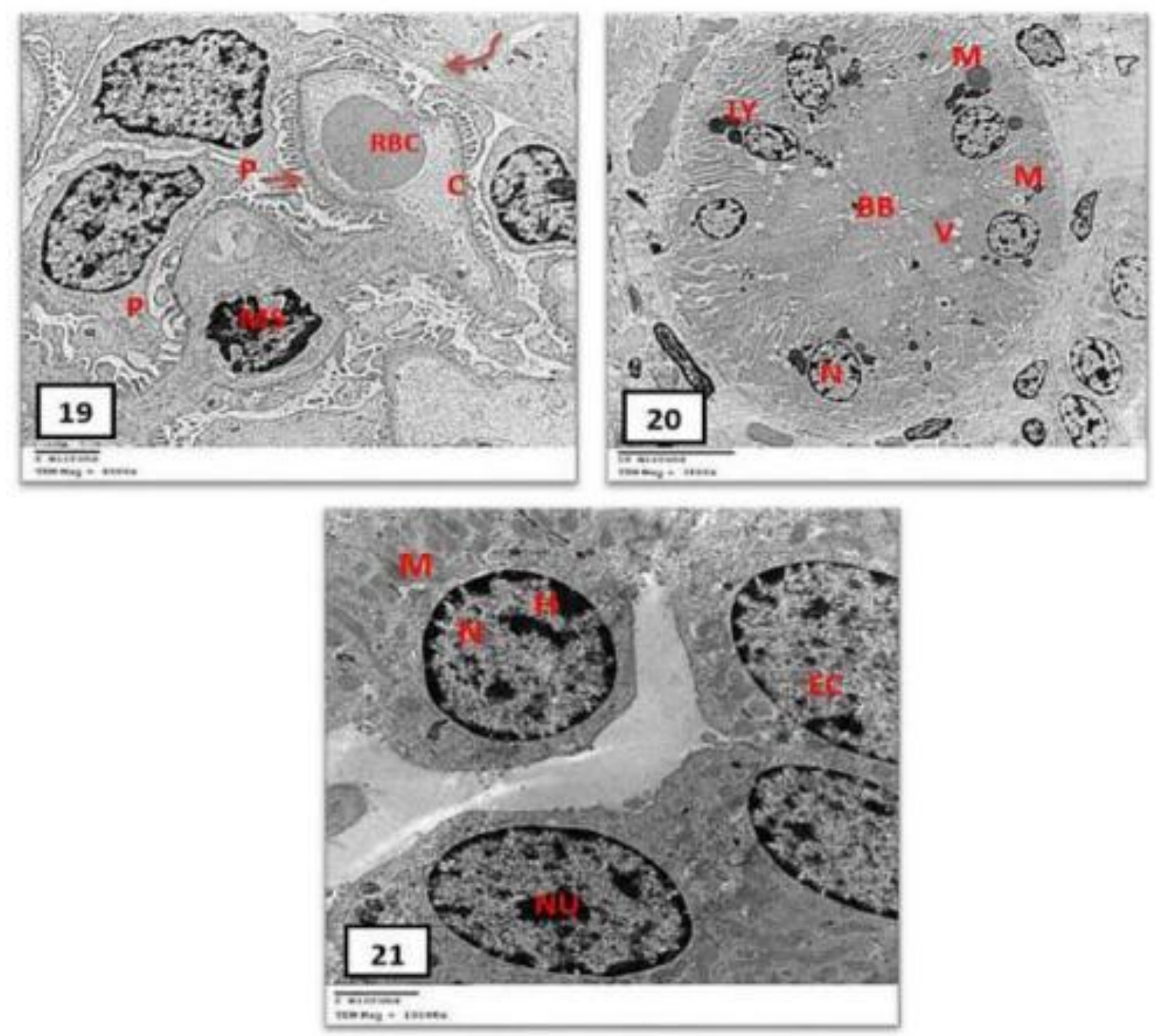

Figures 19-21: electron photomicrograph in sections of the renal cortical tissue of rats in the control group.

Fig. 19: showing normal appearance of renal corpuscle capillaries $(C)$, red blood cell (RBC), glomerular podocytes (P) with their processes (pedicels) $(\rightarrow)$, mesangial cells (MS), and the glomerular basement membrane (broken arrow).

Fig. 20: a proximal convoluted tubule showing normal nucleus $(\mathrm{N})$, mitochondria $(\mathrm{M})$, brush border (BB), lysosome (LY), and membranous vesicles (V).

Fig. 21: a distal convoluted tubule showing a large number of mitochondria (M), few small microvilli, nucleus (N) lying in an apical position normal eccentric nucleolus (NU), homogenous chromatin distribution with euchromatin (EC) and small clumping heterochromatin $(\mathrm{H})$ attached to the inner nuclear envelope. 

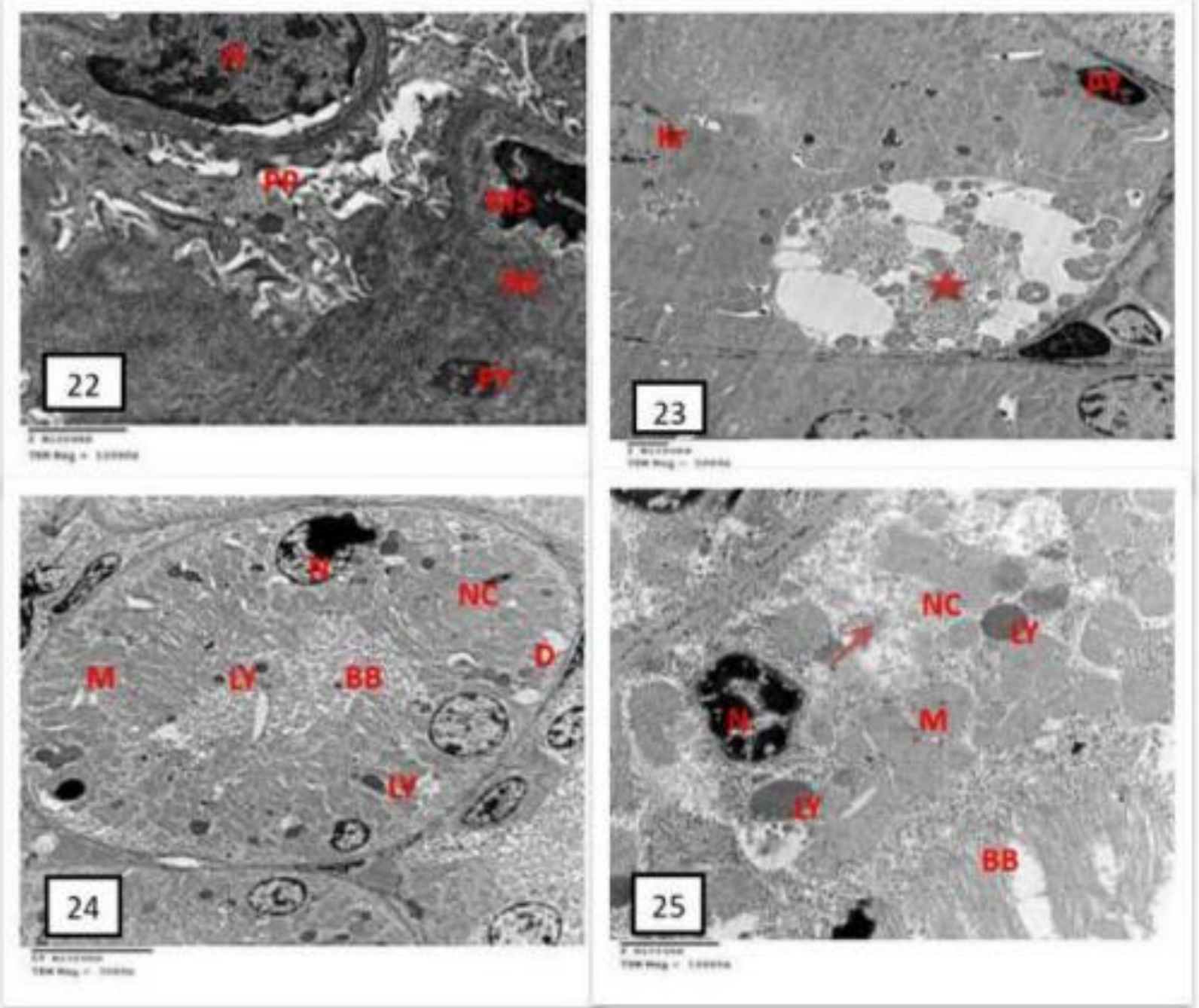

Figures 22-25: electron photomicrograph in sections of the renal cortical tissue of rats in the R groups after 7days of gamma irradiation.

Fig. 22: showing highly corrugated and folded nuclear membrane with densely stained nuclei $(\mathrm{N})$, podocyte processes (PP), and mesangial cells (MS). Notes: necrotic areas (NC) and pyknotic nucleus (PY).

Fig. 23: showing the remnant nuclei are pyknotic (PY) and karyolitic (kr). Note: swelling of the cytoplasm (Star).

Fig. 24: showing densely stained pyknotic nuclei (N), lysosomes (Ly), brush border (BB), increased degenerated (D), and necrotic areas (NC) with swollen of mitochondria (M).

Fig. 25: showing densely stained pyknotic nuclei (N), brush border (BB), lysosomes (Ly), disintegrated mitochondria $(\mathrm{M})$, and necrotic areas (NC) with highly increased collagen deposition $(\rightarrow)$. 


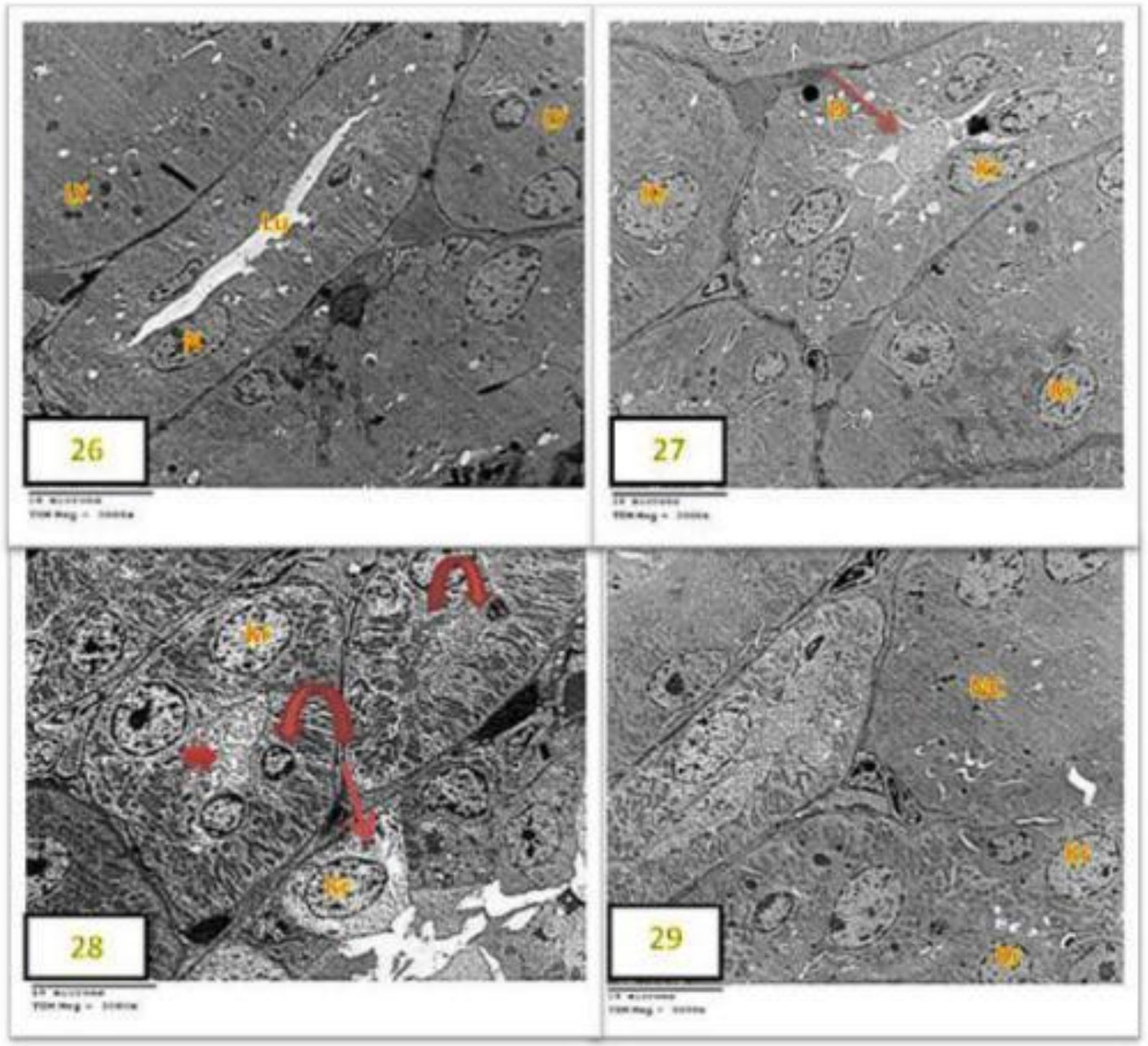

Figures 26-29: electron photomicrograph in sections of the renal cortical tissue of rats in the R groups after 7days of gamma irradiation.

Fig. 26: showing narrow lumen $(\mathrm{Lu})$ with highly reduced number of nuclei $(\mathrm{N})$ of the cuboidal tubules and highly increased lysosomes (LY).

Fig. 27: showing apical parts of the cuboidal cells $(\downarrow)$ with lots of degenerated areas (D) and most nuclei show karyolysis (Kr).

Fig.28: showing completely necrotic cytoplasm $(\downarrow)$ around the karyolitic nucleus (Kr), collagen deposition $(\Rightarrow)$ and some atrophied nuclei ( curved arrow) in the PCT and DCT.

Fig. 29: showing most PCT with necrotic area (NC) and karyolitic (Kr) nuclei. 

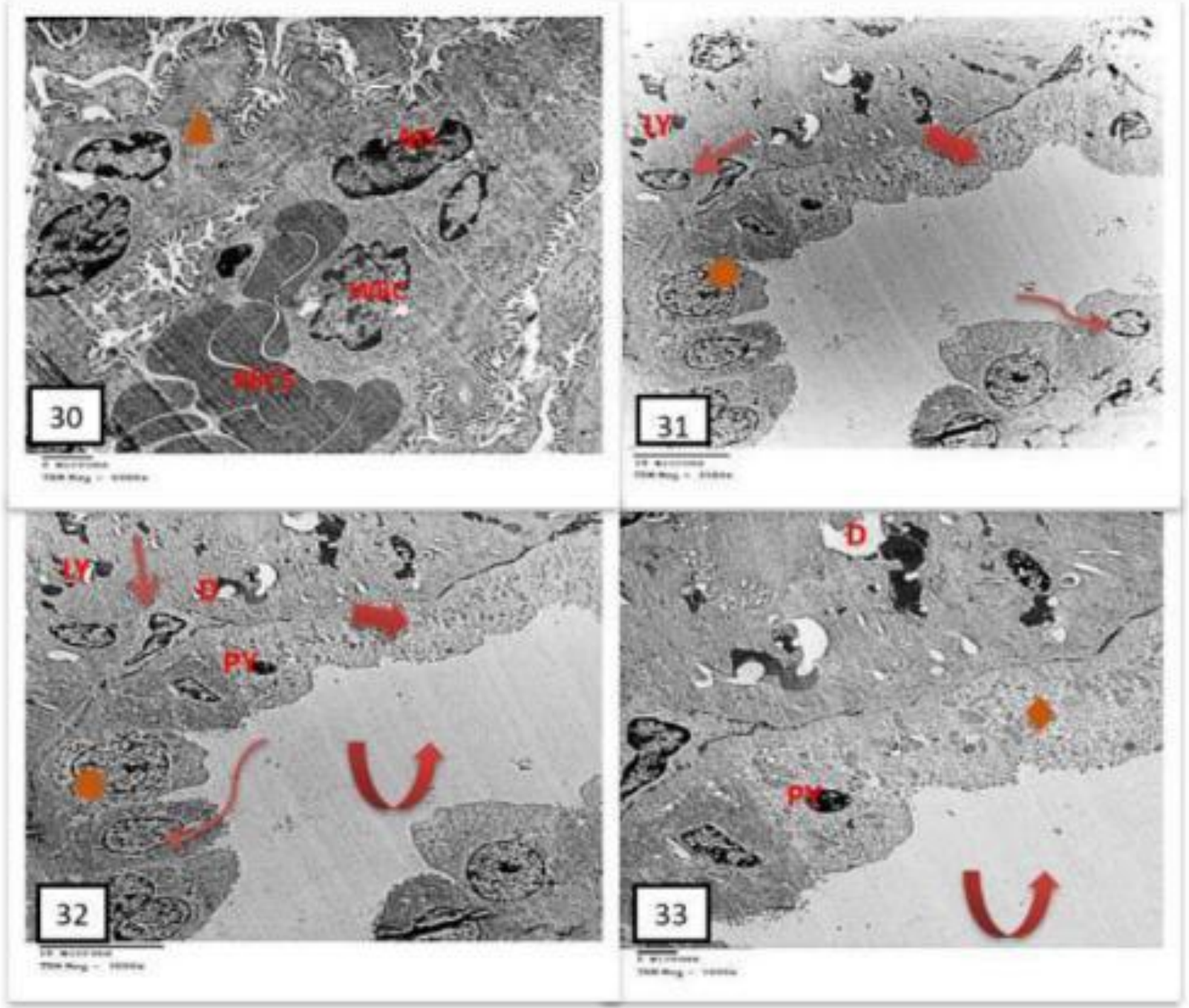

Figures 30-33: electron photomicrographs in sections of the renal cortical tissue of rats in $\mathrm{R}$ groups after 21 days of gamma irradiation.

Fig. 30: showing congested RBCs, malformed nuclei of WBCs, highly thickened podocyte processes of the glomerular tuft $(\mathbf{\Delta})$ and malformed nuclei of the mesangial cells (MS).

Figs. 31-33: DCTs lost their normal architecture because some nuclei of the cuboidal cells contain atrophied (broken arrow) and pyknotic nuclei (PY); others contain nuclei with disintegrated and fragmented chromatin ( $\downarrow$ ). Most of cuboidal cells lost their nuclei $(\downarrow)$ with highly widened lumen (curved arrow). PCT completely lost its normal appearance and appears with debris of degenerated cytoplasmic organoids with unclear border of cuboidal cells; most nuclei appear malformed $(\bullet)$ and brush borders are replaced by collagen fibers deposition (bold arrow $\rightarrow$ ) with numerous lysosomes(LY) and degenerated areas (D). 


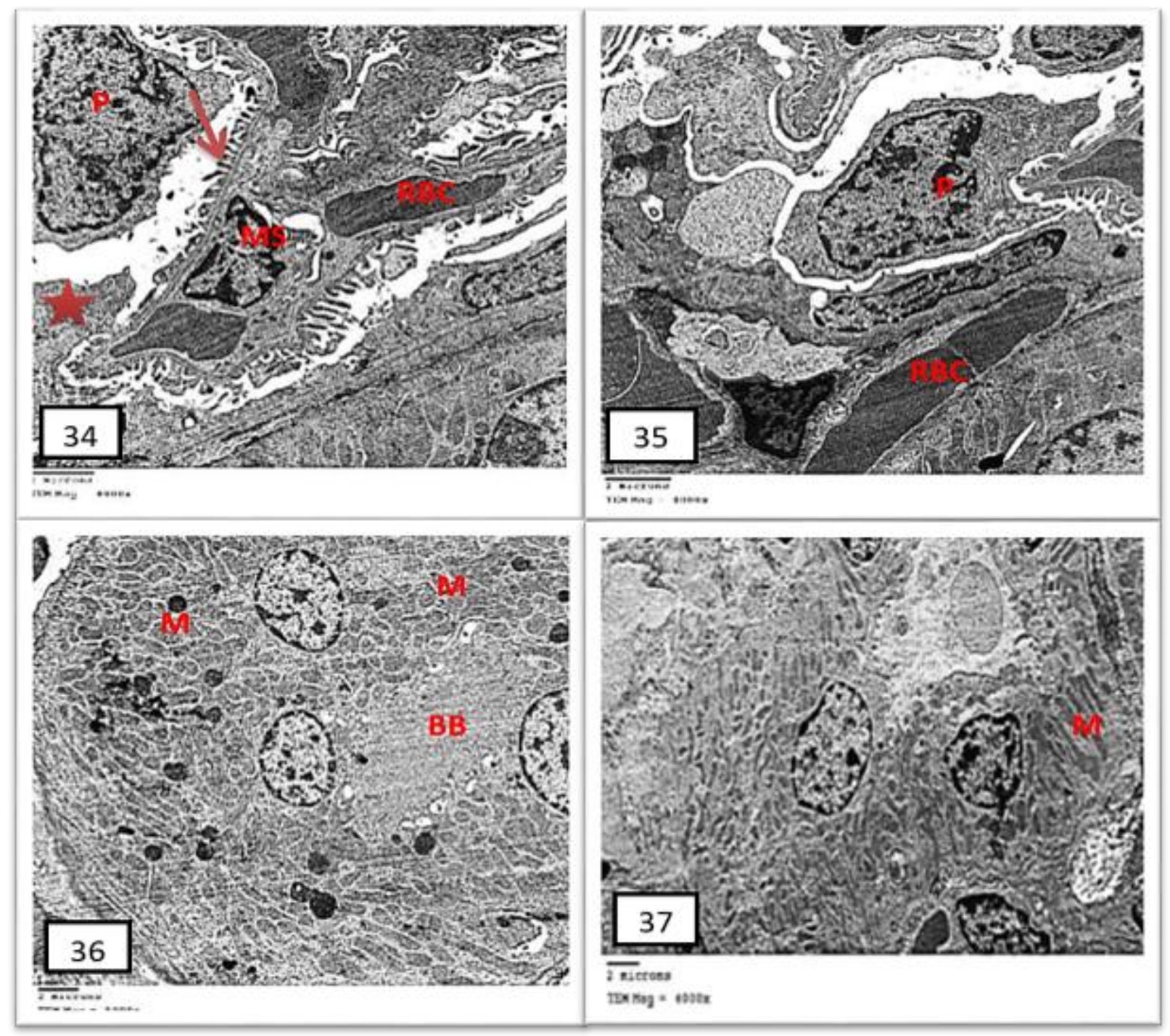

Figures 34-37: electron photomicrograph in sections of the renal cortical tissue of rats in the GA groups after 7 (Figs. 34\&35) and 21 days (Figs. 36\&37) of treatment.

Figs. 34\&35: showing a capillary tuft containing red blood cells (RBC), podocytes (P) with primary (star), secondary $(\downarrow)$ processes and mesangial cells (MS).

Fig.36: PCT showing microvilli brush border (BB) and numerous mitochondria (M)in the basal part of the cell.

Fig. 37: DCT showing large number of mitochondria (M). 

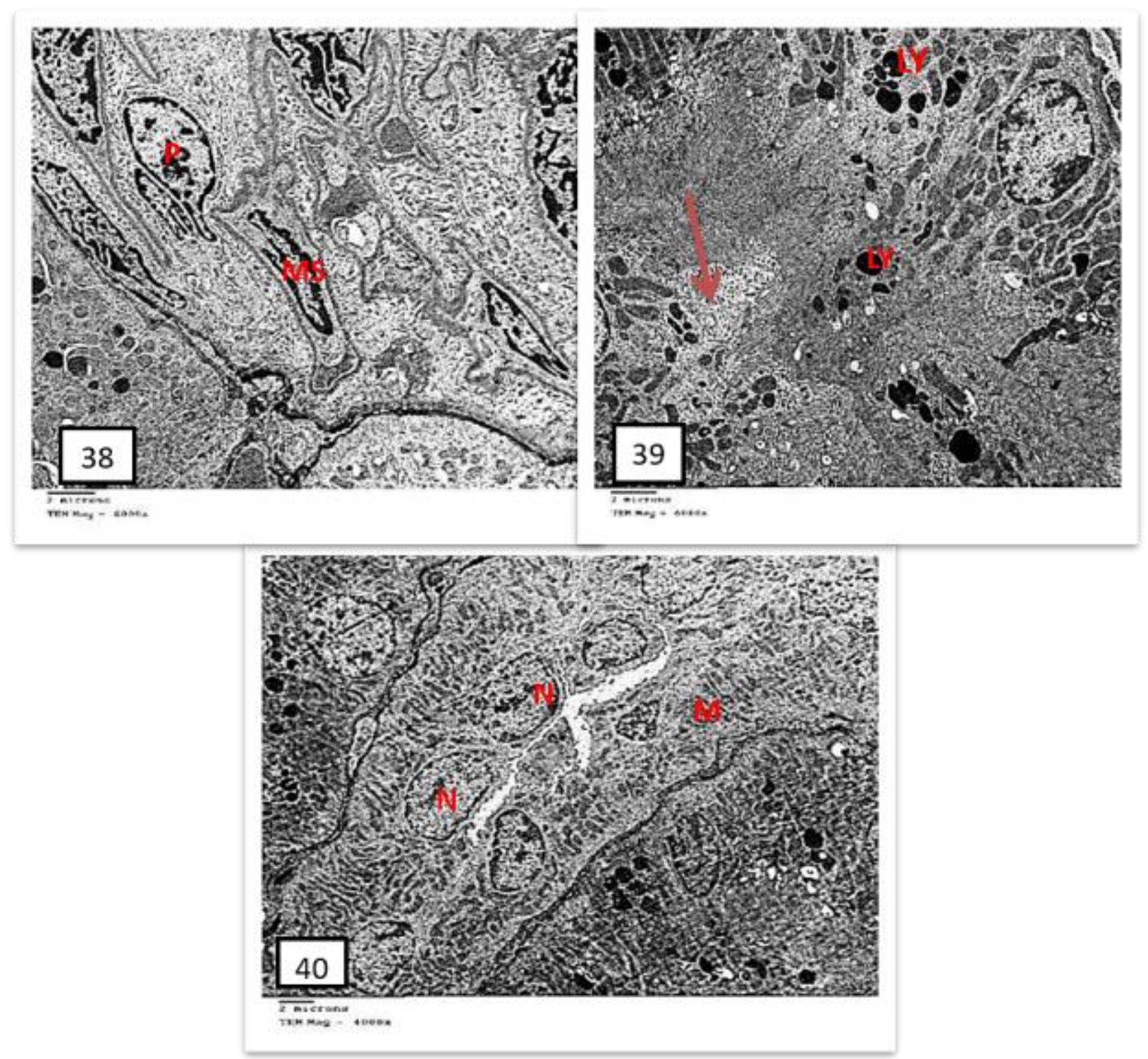

Figures 38-40: electron photomicrograph in sections of the renal cortical tissue of rats inthe GA+R groups after 7 days of irradiation.

Fig. 38: showing normal structure of podocyte (p) and mesangial cells (MS).

Fig. 39: showing somewhat normal appearance of nuclei, brush borders, and mitochondria of cuboidal cells in the PCTs with numerous lysosomes (LY) and collagen fibres deposition $(\downarrow)$ are detected.

Fig. 40: showing somewhat normal appearance of cuboidal cells in the DCTs with well-developed mitochondria (M) and nuclei $(\mathrm{N})$. 

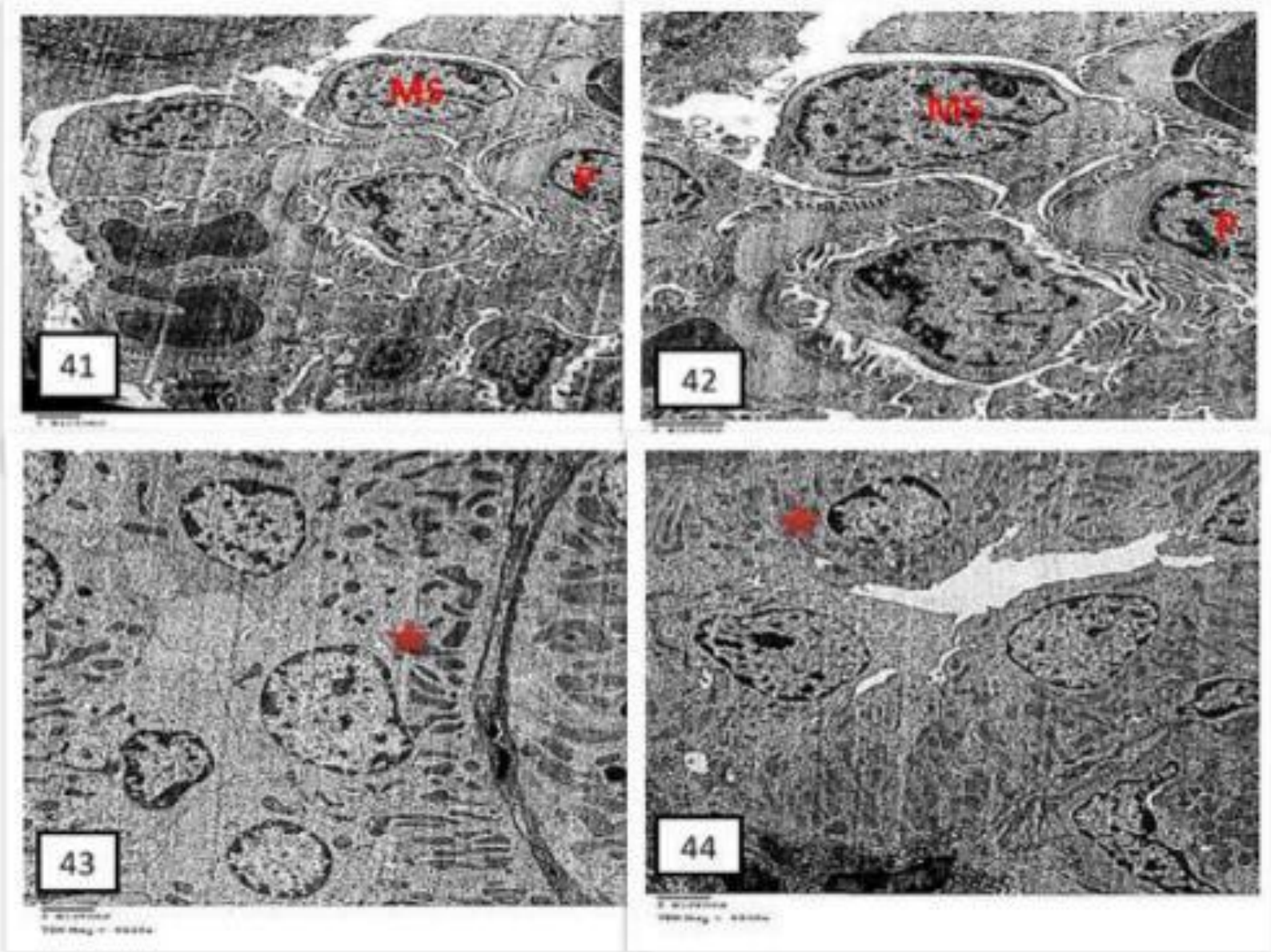

Figures 41-44: electron photomicrograph in sections of the renal cortical tissue of rats inthe GA+R groups after 21 days of irradiation.

Figs. 41\&42: showing normal appearance of mesangial (MS) and podocyte cells (P).

Figs. 43\&44: showing well developed cuboidal cells of PCTs and DCTs with somewhat normal cytoplasmic organoids (star).

\section{DISCUSSION}

Radiotherapy remains an important tool for cancer treatment today and is considered as a part of palliative care because it can also help relieve a patient's symptoms when cancer has spread widely. In some cases, palliative radiation treatment can help to prevent painful tumor effect from developing and improve quality of life by alleviating symptoms such as pain and nausea when the cancer cannot be cured ${ }^{(\mathbf{1 2})}$. The kidney is one of the main radiation dose-limiting organs during the abdominal radiotherapy process and is one of the most radiosensitive organs ${ }^{(13)}$. Radiation is widely used for both diagnosis and therapy and chronic oxidative stress is one of the most important side effects of whole-body irradiation that causes radiation nephropathy ${ }^{(\mathbf{1 4})}$. Single low-doses of 4.5-6.0 Gy may induce chronic renal damage ${ }^{(15)}$. Kidney tubular cells are one of the most important sites for radiation damage ${ }^{(\mathbf{1 6})}$.
Present observations on gamma radiation-treated rats after both histopathological and ultrastructural examination of sections of the kidney cortical tissue 7 and 21 days revealed several changes such as highly affected cortical glomeruli and highly altered architecture of the PCTs and DCTs. Similar alterations were previously reported after gamma radiation exposure ${ }^{(17,18)}$. These changes could be caused by the release of different free radicals and ROS ${ }^{(19)}$, thus enhancing oxidative damage and obliteration of the kidney cortical cellular constituents. Impaired amino acid and glucose reabsorption can result from its alterations because the brush border membrane plays an important role in proximal tubular reabsorption processes ${ }^{(20)}$. The rupture of PCT brush border and degenerated mitochondria are among the critical findings after radiation exposure; these ultrastructural changes were described earlier ${ }^{(21)}$, where the same authors 
attributed brush border rupture to the degenerative changes of the whole tubules and mitochondrial degeneration to increased water influx that leads to inner and outer mitochondrial chamber membrane separation and finally their degeneration. In addition, the increase in lysosomal bodies and chromatin condensation of the renal cortical tissue after radiation exposure throughout the irradiation period could be indicative of cellular degenerative activity ${ }^{(22)}$. Increased deposition of collagen fibers after exposure to $5 \mathrm{~Gy}$ of gamma radiation is one of the most important findings in this experiment; such observation was also previously observed $^{(23)}$. Renal tissue fibrosis was reported earlier to progress to end in renal failure ${ }^{(24)}$. The fibrosis process comprises 4 stages, namely, priming, activation, execution and progression. Radiation exposure is a priming agent that produces activation of fibrogenic cells, including cortical interstitial fibroblasts. Upon activation, such fibrogenic cells enhance the collagen production process ${ }^{(25)}$. In the same direction, oxidative stress was reported to stimulate expression of collagen biosynthesis genes, thus increasing collagen fibers deposition (26). Exposure to IR is associated with excessive ROS production (27); ROS generation rate imbalance induces oxidative stress and subsequent production of free radicals that can destroy DNA, proteins, and lipids ${ }^{(28)}$. The lipid peroxidation directly attacks phospholipids and can initiate cell death. Oxidized phospholipids can induce inflammatory disease and cellular pro-inflammatory changes ${ }^{(29)}$. Mitochondrial DNA degradation could also be caused by ROS accumulation ${ }^{(30)}$. Therefore, most of the current histopathological changes in the renal tissue after gamma radiation exposure could be attributed to the liberation of free radicals and their subsequent interaction with the cellular membrane polyunsaturated fatty acids of their phospholipids portion ${ }^{(31)}$. The findings of this experiment revealed that GA can effectively act as an ameliorative agent. Notably, 21 days of treatment with GA in the irradiated rats diminished the drastic effects of radiation exposure, which is indicated by restoration of the normal kidney cortical architecture where the Bowman's capsules appeared intact with mesangial cell proliferation and normal collagen fiber deposition throughout the cortical tissue. PCTs cells showed multiple signs of regeneration, such as rounded nuclei and brush border regeneration with polymorphic lysosomes whereas DCTs retained their microvilli and rounded nuclei. GA acts as a potent antioxidant that scavenges free radicals to prevent or ameliorate the toxic effects of gamma radiation, as shown in the histopathological and ultrastructural changes and might provide substantial protection against radiation-induced damage.GA has been used long time ago to treat chronic renal failure disease ${ }^{(32)}$; it was formerly reported to have a positive effect against inflammation and oxidative stress in animals ${ }^{(33)}$ and patients with chronic kidney disease ${ }^{(34)}$. It was reported earlier to counteract lipid peroxidation effects by decreasing malondialdehyde and superoxide production, increasing glutathione and total antioxidant capacity in nephrotoxicity-induced and chronic renal failure rats ${ }^{(35)}$. In addition, it is described as a human anti-oxidative agent that increases the total antioxidant capacity level and decreases oxidative stress markers in patients with sickle cell anemia ${ }^{(36)}$. Its antioxidant effect could be caused by its amino acids ${ }^{(37)}$, such as hydroxyproline, proline and aspartic acid ${ }^{(32)}$.GA also has immunemodulatory and anti-inflammatory effects ${ }^{(38)}$.

\section{CONCLUSION}

In this experiment, GA ameliorated most of the damaging effects of the rats cortical tissues after radiation exposure. Therefore, because it overcomes the side effects that might accompany gamma radiation exposure, one would recommend the administration of $\mathrm{GA}$ as a radio protective agent.

\section{REFERENCES:}

1. Ping $Z$, Peng $Y$, Lang $H$ et al. (2020): Oxidative stress in radiation-induced cardiotoxicity. Oxid. Med. Cell Longev., $7: 1-15$.

2. Mavragani I, Nikitaki Z, Kalospyros S et al. (2019): onizing radiation and complex DNA damage: From prediction to detection challenges and biological significance. Cancer, 11(11):1-29.

3. Tan B, Norhaizan M, Liew W et al. (2018): Antioxidant and oxidative stress: a mutual interplay in age-related diseases. Front. Pharmacol., 24(9):1-28.

4. Przystupski D, Górska A, Rozborska P et al. (2019): The cytoprotective role of antioxidants in mammalian cells under rapidly varying UV conditions during stratospheric balloon campaign. Frontiers Pharmacology, 851(10):1-19.

5. Said M A, Atwa S, Khalifa O (2019): Ameliorating effect of gum Arabic and lemongrass on chronic kidney disease induced experimentally in rats. Bull. Nat. Res. Cent., 43(47):1-8.

6. Dauqan E, Abdullah A (2013): Utilization of gum Arabic for industries and human health. Ame. J. App. Sci., 10:1270-1279.

7. Mohammed A (2017): Estimation of the active components in gum Arabic collected from western Sudan. Int. J. Sci. Res., 6:1262-1282.

8. Ali B, Al-Salam S, Al Husseni I et al. (2010): Effects of gum Arabic in rats with adenine-induced chronic renal failure, Exp. Biol. Med., 235(3):373-382. 
9. Gamal El-din A, Mostafa A, Al-Shabanah O et al. (2003):Protective effect of Arabic gum against acetaminophen-induced hepatotoxicity in mice. Pharmacol. Res.,48: 631-635.

10. Bancroft J, Gamble $M$ (2008): Connective tissues and stains. In: Theory and Practice of Histological Techniques. $5^{\text {th }}$ ed. Elsevier Health Science, London, Pp: 135-160.

11. Mercer E, Birbeck M (1966): Electron microscopy. In: A Hand Book for Biologists. $2^{\text {nd }}$ ed. Blackwell Scientific Publications. Oxford, Pp:102-109.

12. Spencer K, Parrish R, Barton $R$ et al. (2018): Palliative radiotherapy. BMJ., 3: 1-12.

13. Mehrvar S, La Cour M, Medhora M et al. (2019):Optical metabolic imaging for assessment of radiation-induced injury to rat kidney and mitigation by lisinopril. Ann. Biomed. Eng., 47(7):1564-1574.

14. Ozbek E (2012): Induction of oxidative stress in kidney. Int. J. Nephro., 2012:1-9.

15. Moulder E, Cohen E (2005): Radiation-induced multiorgan involvement and failure: the contribution of radiation effects on the renal system. Bri. J. Radio., 27(1):82-88.

16. Soranson J, Denekamp J (1986): Precipitation of latent renal radiation injury by unilateral nephrectomy. Br. J. Cancer, 53(7):268-272.

17. Kanter M, Topcu-Tarladacalisir Y, Uzal C (2011):Role of amifostine on acute and late radiation nephrotoxicity. Int. J. Exp. Clini. Pathophysio. Drug Res., 25(1):77-85.

18. Mansour H, Abd-El Azeem M, Ismael N (2014): Protective effect of moringaoleifera on $\gamma$ - radiationinduced hepatotoxicity and nephrotoxicity in rats. Ame. J. Phytom. Cli. Ther., 2(4): 495-508.

19. Giardi M, Touloupakis E, Bertolotto D et al. (2013): Preventive or potential therapeutic value of nutraceuticals against ionizing radiation-induced oxidative stress in exposed subjects and frequent fliers. Int. J. Mol. Sci., 14:17168-17192.

20. Kim Y, Byun H, Kim Y et al. (1995): Effect of cisplatin on renal-function in rabbits: mechanism of reduced glucose reabsorption. Tox. App. Pharmaco., 130(1):19-26.

21. Abu-Nour S, Rady M, Khalil I (2008): Protective effect of $\beta$-carotene on gamma radiation- induced renal histological and ultrastructural changes in male albino rats. Al-Azhar Bulletin of Science, 19(1): 27-44.

22. Kamble P, Kulkarni S, Bhiwgade D (2013): Ultrastructural and antioxidant studies of etoposide treated kidney of rat. J. Cancer Sci. Ther., 5(4):137-141.

23. Parker G, Cohen E, Li N et al. (2019): Radiation nephropathy in a nonhuman primate model of partial-body irradiation with minimal bone marrow sparing-part 2: histopathology, mediators, and mechanisms. Health Physics, 116(3):409-425.
24. Cohen E (1995): Fibrosis causes progressive kidney failure. Medical Hypotheses, 45(5):459-462.

25. Liu D, Wang T (2008): Role of connective tissue growth factor in experimental radiation nephropathy in rats. Chinese Medical Journal, 121(19): 1925-1931.

26. Guler G, Turkozer Z, Ozgur E et al. (2009): Protein oxidation under extremely low frequency electric field in guinea pigs. Effect of N-acetyl-L-cysteine treatment. Gen. Physiol. Biophys., 28(1):47-55.

27. Srinivas U, Tan B, Vellayappan B et al. (2018): ROS and the DNA damage response in cancer. Redox Biology, 25:101084-88.

28. Que X, Hung H, Yeang $C$ et al. (2018): Oxidized phospholipids are pro-inflammatory and pro-atherogenic in hypercholesterolaemic mice. Nature, 558: 301-306.

29. Latunde-Dada $G$ (2017):Ferroptosis: Role of lipid peroxidation, iron and ferritinophagy. Bioch. Et. Biophysica. Acta. General Subjects, 1861(8): 1893-1900.

30. Shokolenko I, Venediktova N, Bochkareva A et al. (2009): Oxidative stress induces degradation of mitochondrial DNA. Nucleic Acids Res., 37(8):2539-2548.

31. Zhao W, Robbins $M$ (2009): Inflammation and chronic oxidative stress in radiation-induced late normal tissue injury: therapeutic implications. Cur.Med. Che., 16(2):130143.

32. Ali B, Ziada A, Blunden G (2009): Biological effects of gum Arabic: a review of some recent research. Food Chem. Toxic., 47(1):1-8.

33. Ali B, Al-Husseni I, Beegam S et al. (2013): Effect of gum Arabic on oxidative stress and inflammation in adenineinduced chronic renal failure in rats. PLoS One, 8(2):55242.55250 .

34. Elamin S, Alkhawaja M, Bukhamsin A et al. (2017): Gum Arabic reduces C-reactive protein in chronic kidney disease patients without affecting urea or indoxyl sulfate levels. Int. J. Nephro., 2017(8):1-6

35. Al-Majed A, Abd-Allah A, Al-Rikabi A et al. (2003): Effect of oral administration of Arabic gum on cisplatininduced nephrotoxicity in rats. J. Biochem. Mol. Toxic., 17(3):146-153.

36. Kaddam L, Fadl-Elmula I, Eisawi O et al. (2017): Gum Arabic as novel anti-oxidant agent in sickle cell anemia phase II trial. BMC. Hem., 17(4): 1-16.

37. Rodrigues E, Mariutti L, Faria A et al. (2012): Microcapsules containing antioxidant molecules as scavengers of reactive oxygen and nitrogen species. Food Chem., 134(2):704-711.

38. Kamal E, Kaddam L, Dahawi M et al. (2018): Gum Arabic fibers decreased inflammatory markers and disease severity score among rheumatoid arthritis patients, phase II trial. Int. J. Rheum., 2018:1-6. 